\title{
Surface Impedance Modeling Using the Finite-Difference Time-Domain Method
}

\author{
David V. Thiel, Senior Member, IEEE, and Raj Mittra, Life Fellow, IEEE
}

\begin{abstract}
The finite-difference time-domain (FDTD) technique has been used to model the one-dimensional (1-D) surface impedance of a lossy earth plane having discontinuities in two and three dimensions. Using a horizontal magnetic field aperture source located five cells from an absorbing boundary and 35 cells above the lossy earth plane, the surface impedance was accurately modeled at a distance of $\lambda_{0} / 5000$ from the source using both grazing and normal incidence. The technique was validated by comparison with a number of two-dimensional (2-D) analytical models. The surface impedance profile in the vicinity of a vertical conductive water filled shaft that extends from the earth's surface to a conductive basement is presented. Unlike modeling in the frequency domain, a single FDTD solution yields accurate multi frequency surface impedance data providing a number of standard cell size constraints are met. For common earth electrical constants, the FDTD approach is limited to frequencies above $500 \mathrm{~Hz}$.
\end{abstract}

Index Terms - Electromagnetic geophysics, FDTD, surface impedance, 3-D modeling, VLF EM.

\section{INTRODUCTION}

$\mathbf{S}$ URFACE impedance measurements are used to map the conductivity structure of the upper parts of the earth's subsurface. Since the 1950's, when the early work related to only the magnetotelluric method [1]-[3], this broad classification has been used across the frequency range from $20 \mathrm{kHz}$ to $10^{-4} \mathrm{~Hz}$. Both naturally occurring radiation sources, which includes the magnetotelluric method (MT), the audiomagnetotelluric method (AMT), and atmospherics based techniques [4], and artificial sources which include controlled source audiomagnetotellurics (CSAMT) [5], very low frequency (VLF) [6], and other radio sources (RMT) [7] are now used. The basic premise underlying the technique is that the position of measurement lies in the far field of the radiation source so that all forward modeling and inversion techniques assume plane wave interactions. In the case of MT, the plane wave is assumed to be incident from the vertical and with the other techniques, the radiation is incident at grazing incidence (e.g., AMT, CSAMT, atmospherics, VLF, and RMT). These latter techniques all operate at frequencies higher than $500 \mathrm{~Hz}$ and all use multifrequency measurements and a one dimensional (scalar) measurement method. Thus, the surface impedance measurement is made with the electric field parallel to the

Manuscript received May 23, 1995; revised September 27, 1996.

D. V. Thiel is with the School of Microelectronic Engineering, Griffith University, Queensland, Australia 4111 (e-mail: dthiel@me.gu.edu.au).

R. Mittra was with the Electromagnetic Communications Laboratory, University of Illinois, Urbana, IL 61801 USA. He is now with the Department of Electrical Engineering, Pennsylvania State University, University Park, PA 16802 USA (e-mail: r1mece@engr.psu.edu).

Publisher Item Identifier S 0196-2892(97)04104-1. plane of incidence. In this paper, only one dimensional measurements will be considered.

At low frequencies, in most environments, conduction currents in the earth dominate the displacement currents. The one dimensional surface impedance, $Z_{s}$, is independent of the vertical angle of incidence and is given by

$$
Z_{s}=\frac{E_{y}}{H_{x}}
$$

where $E_{y}$ is the horizontal electric field component measured in the plane of incidence and $H_{x}$ is the horizontal magnetic field component measured perpendicular to the plane of incidence.

If the lossy earth overlies a perfect conductor at a depth $h$, then the surface impedance $Z s_{1}$ is given by the transmission line equation with a short circuit termination [8]:

$$
Z s_{1}=Z_{s} \tanh (\gamma h)
$$

where $\gamma$ is the complex propagation constant for the medium, i.e.,

$$
\gamma^{2}=j \sigma \mu \omega-\varepsilon \mu \omega^{2}
$$

and $\sigma$ is the conductivity, $\omega$ is the angular frequency of the radiation, $\varepsilon$ is the dielectric permittivity, and $\mu$ is the magnetic permeability of the earth and $j=\sqrt{-1}$.

Given the complexities of the earth's subsurface including horizontal layering, vertical faults, and dislocations and folded structures, the forward modeling problem using analytical methods is limited to a few idealized cases [9]. With the advent of numerical modeling techniques applied to the field of geophysics, a number of different techniques have been used to model a three-dimensional (3-D) earth plane. In particular, the finite element method [10], [11], and integral equation methods [12]-[14] have been successfully employed. The great utility of the finite difference time domain (FDTD) method [15] in solving a wide variety of problems in the fields of radar [16], antenna modeling [17], and more recently in electromagnetic geophysics with near field sources [18], has prompted this investigation into FDTD modeling of the surface impedance method. The primary purpose of this paper is to assess the utility of the FDTD method in surface impedance forward modeling.

Three-dimensional modeling of surface impedance in the frequency domain at ULF frequencies, has been undertaken previously by Mackie and Madden [19], [20], who developed a difference scheme for the quasistatic solution to Maxwell's equations in integral form. Their formulation results in cells of $E$ and $H$ offset by one half a cell just as is the case for Yee's FDTD method [15]. The source employed was a 
uniform current sheet at the earth's surface with a number of air layers above to ensure secondary fields are accommodated in the solution. Wang and Hohmann [21] employed a FDTD approach to solve for the 3-D earth response due to a finite source field; i.e., a vertical magnetic dipole and also a current loop lying on the earth's surface. The emphasis in our work is to employ plane wave excitation rather than a near field source, at much higher frequencies.

The most important difficulty encountered by numerical methods in modeling surface impedance is the application of the source in the far field and the imposition of absorbing boundary conditions (ABC's) at the terminations of the solution space, both above the earth's surface and also beneath it. In addition, most surface impedance measurements are made across a broad range of frequencies. Solutions in the frequency domain must be run at each frequency separately. In FDTD, these problems can be solved in a manner that does not require the solution of a matrix set that is so large as to preclude solution in a realistic time. These limitations are explored in addition to the presentation of some results for two and three dimensional earth models.

\section{FDTD MODELING CONSTRAINTS}

The FDTD method is a numerical method of solving Maxwell's equations directly by discretizing the solution volume or area by a regular mesh. The electric field is calculated from surrounding magnetic field values and the magnetic field is solved from the adjacent electric field values. This calculation is performed for every node in the solution space at time $t=0$, and then for $t=\Delta t, t=2 \Delta t$, etc. The source field is usually in the form of a pulse of finite width in time activated along a line in two-dimensional (2-D) and a plane in 3-D modeling. In allowing this pulse to propagate throughout the solution space, one can convert the transient field at any point to the field as a function of frequency using a discrete Fourier transform. Thus, the solution of the problem in time allows one to extract frequency information over a relatively broad range. This technique is used through out this paper.

The solution space will have boundaries at its extremities, and these boundaries must interact with the fields in predictable ways. A perfect electric conducting boundary (PEC) will force the electric field to be perpendicular to the boundary, and a perfect magnetic conducting boundary (PMC) will force the magnetic field to be perpendicular to the boundary. To minimize reflection at the boundary, we must use an absorbing boundary condition (ABC). There is significant discussion in the literature about how this can best be achieved. In this work, a second order Mur ABC [22] was employed and was found to be satisfactory.

As with all numerical modeling techniques, the accuracy of the solution is constrained by the division of the solution space into cells; in this solution the cells are a uniform array of rectangular bricks. Any structure to be modeled must be divided into this regular array of cells $(\Delta x \times \Delta y \times \Delta z)$, with the three dimensions being within an order of magnitude of each other.

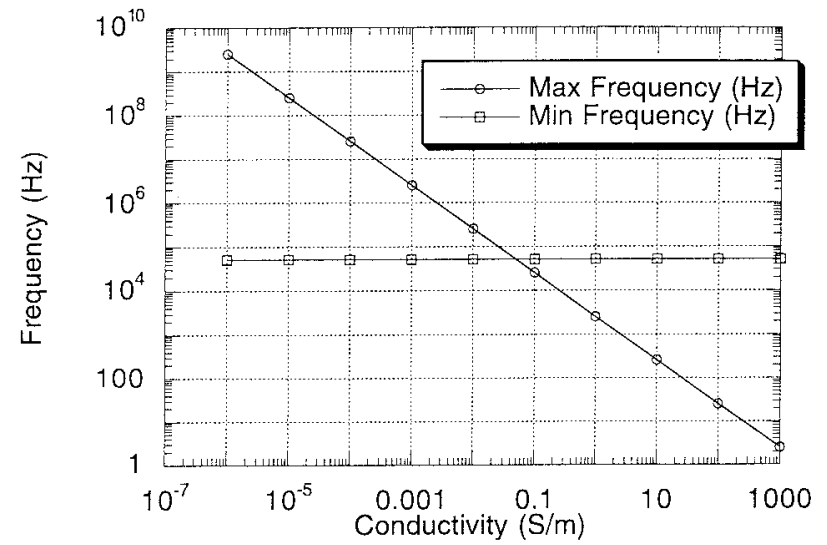

Fig. 1. Limiting conditions for the FDTD method in lossy media. The maximum frequency has been calculated from the skin depth, and the minimum frequency was derived from Courant condition with $N=10^{4}$ time steps and $\Delta x=\Delta y=\Delta z=1 \mathrm{~m}$. Valid FDTD solutions are found between the maximum and minimum frequencies.

The solution of any problem using the FDTD method is only accurate and stable if the time step $\Delta t$ is constrained by the Courant condition, i.e.,

$$
\Delta t \leq\left\{\frac{\operatorname{Min}\left(\mu_{r}\right) \operatorname{Min}\left(\varepsilon_{r}\right)}{c^{2}\left[(\Delta x)^{-2}+(\Delta y)^{-2}+(\Delta z)^{-2}\right]}\right\}^{1 / 2}
$$

where $c$ is the speed of light in a vacuum, and $\operatorname{Min}\left(\mu_{r}\right)$ and $\operatorname{Min}\left(\varepsilon_{r}\right)$ are the minimum values of the relative permeability and the relative permittivity, respectively. Sampling in the frequency domain is determined from the Fourier transform of the time transient at any point in the solution space. Thus, the smallest frequency resolution $\Delta f$ is given by

$$
\Delta f=\frac{1}{N \Delta t}
$$

where $N$ is the number of time steps used in solving the model.

When dealing with a lossy material, accurate solutions are obtained only when the cell size is less than $10 \%$ of the skin depth of the medium $\delta$ where $\delta=1 / \sqrt{j \pi \mu \sigma f}$ and $f$ is the frequency of the radiation. Only then can the computational model accommodate the rapid changes in phase and amplitude of the fields as they propagate. Note that the wavelength in a lossy material is $2 \pi \delta$. The sensitivity of the surface impedance method is limited to a depth of approximately one skin depth, and so the minimum number of cells beneath the surface of the earth to model an infinitely deep uniform earth is 10 . For multi frequency investigations, this calculation must be performed at the highest frequency of interest. Consequently, to obtain a frequency profile covering two orders of magnitude, the earth plane must be modeled as 100 cells deep. This effect, however, can be minimized if one is interested in modeling structures that are shallow, providing an effective absorbing boundary condition can be applied or a highly conductive basement is used.

The limiting conditions given by (3) and (4) can be combined as the inequality that gives the limiting conditions on $f$

$$
\frac{c \Delta x_{\min } 3^{1 / 2}}{N}<f<\left(100 \pi \mu_{0} \sigma \Delta x_{\min }^{2}\right)^{-1}
$$




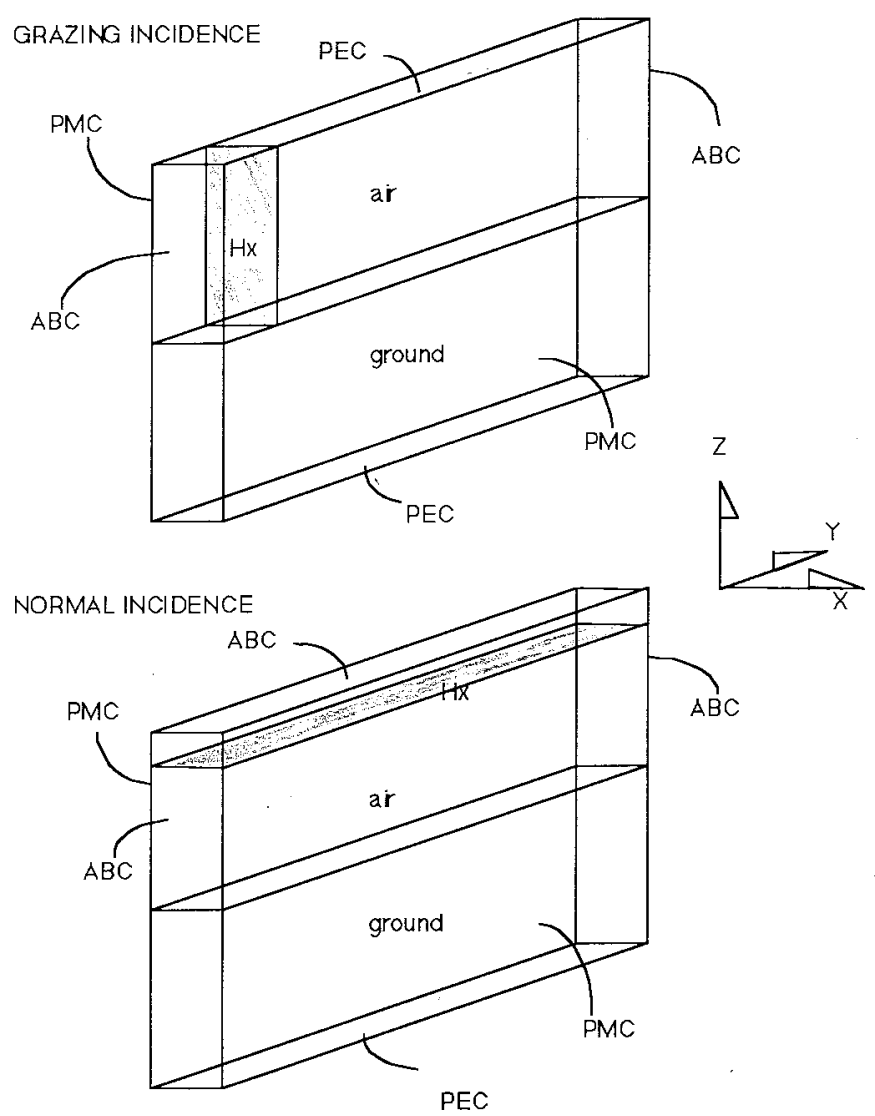

Fig. 2. Two dimensional modeling volume showing the source configuration and boundary conditions for normal incidence and grazing incidence, together with the coordinate system $(n x=1, n y=100, n z=40)$.

assuming cubic cells and $\mu_{r}=1=\varepsilon_{r}$. These two functions are plotted together in Fig. 1 , using $\Delta x_{\min }=1 \mathrm{~m}$ and $N=10^{4}$. There is a point of intersection where the maximum frequency is equal to the minimum frequency. This point is dependent on both $N$ and $\Delta x_{\min }$, and occurs at a particular conductivity value. Accurate solutions will be obtained only when this inequality is met, i.e., the model uses conductivity values less than this cross-over value. It will be demonstrated in this paper that such conditions can be relaxed for surface impedance calculations without loss of solution accuracy. Of particular significance to the modeling of surface impedance relevant to geophysical investigation is the range of frequencies for which a valid solution can be obtained. From Fig. 1, it is noted that if the earth conductivity is $0.001 \mathrm{~S} / \mathrm{m}$, then valid solutions are only obtained in the frequency range $40 \mathrm{kHz}-2$ $\mathrm{MHz}$. Given that common earth conductivities are found in the range of $10^{-5}-10 \mathrm{~S} / \mathrm{m}$, and the frequencies of interest in MT lie in the range $0.001-1000 \mathrm{~Hz}$, one must choose a value of $\Delta x_{\min }=500 \mathrm{~m}$ and $N=3 \times 10^{14}$ time steps. A conventional FDTD solution in 3-D would be extremely computationally intensive. For this reason, the modeling undertaken in this paper relates to a higher frequency regime.

In this paper, all modeling was conducted at relatively high frequencies, $10^{4}-10^{6} \mathrm{~Hz}$ using a cell size of $1 \mathrm{~m}$. This is directly relevant to the VLF, and RMT surface impedance methods.

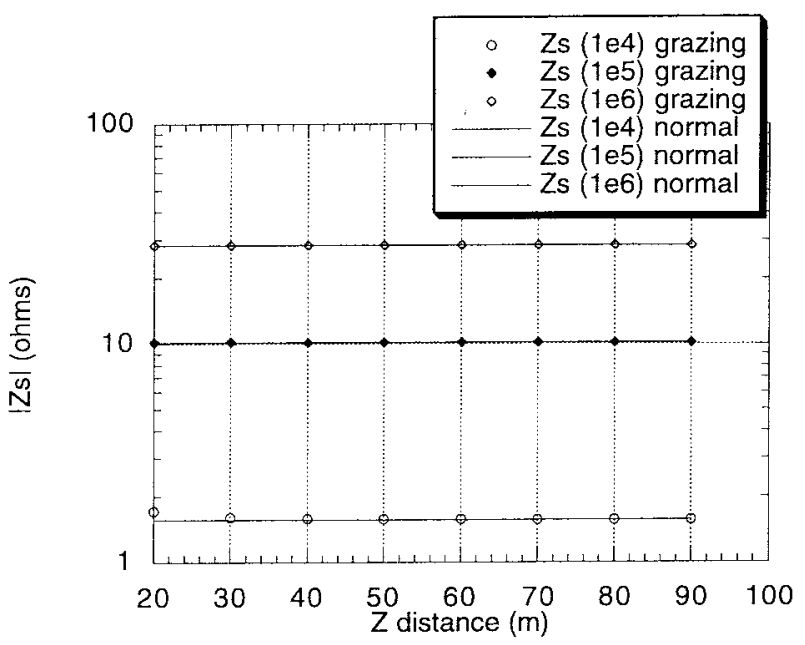

(a)

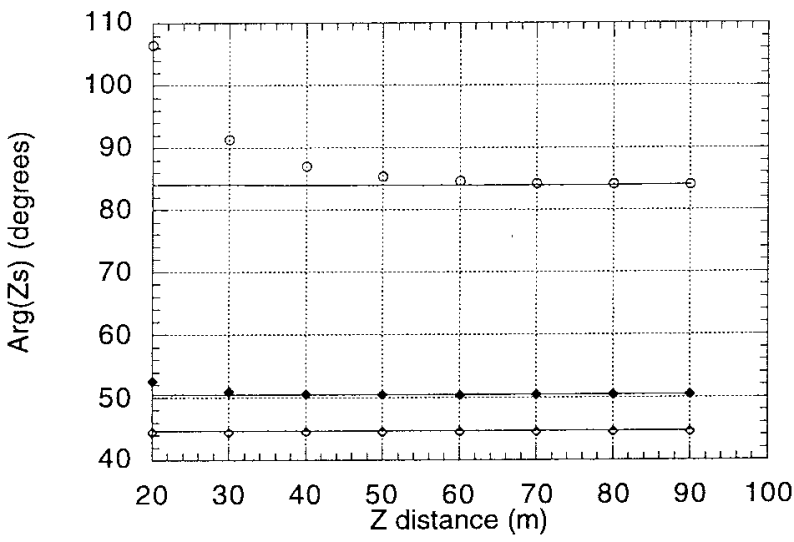

(b)

Fig. 3. Variation of surface impedance across the solution space for the two different excitation sources illustrated in Fig. 2 (continuous line-normal incidence, circles-grazing incidence). The model is a layered earth (upper layer: depth $20 \mathrm{~m}$, conductivity $0.01 \mathrm{~S} / \mathrm{m}$, lower layer is a perfect conductor). The surface impedance was calculated using $E_{y}$ at $z=20$ and $H_{x}$ at $z=21$. $(N=10000)$.

The proximity of the source to the solution space under consideration can be considerably less than a wavelength if one employs an impressed plane wave field. This minimizes the size of the total grid. Four different sources were investigated with the $H$ field component parallel to the vertical discontinuity (referred to as $H$ field or TM mode investigations): vertical sheets of $H_{x}$ and $E_{y}$, which corresponds to grazing incidence, and horizontal sheets of $H_{x}$ and $E_{z}$, which correspond to normal incidence. The $H$ field sources were found to give a larger valid solution space than that obtained using an $E$ field source. Note that the use of ABC's is minimized, not only because they are ineffective at grazing incidence, but also because the computation time is significantly increased. The use of perfect magnetic conductors (PMC's) perpendicular to the magnetic field source and PEC's parallel to the magnetic field source ensure uniform plane wave propagation conditions throughout the solution space.

\section{Two Dimensional Modeling}

All 2-D modeling was undertaken using the FDTD code TAP Version 3.25 developed at the Electromagnetic Commu- 
TABLE I

Comparison of 2-D Model Surface Impedance Results With Theory. In THE FDTD MOdel, THE LoSSY EARTH (CONDUCTIVITY $=0.01 \mathrm{~S} / \mathrm{m}$ AND $0.001 \mathrm{~S} / \mathrm{m}$ ) was $20-\mathrm{m}$ Thick Overlying a Perfect Conductor. The Multilayer Solution Takes the PEC Into Account, Whereas the Infinite Solution Assumes an Infinite Depth of Lossy Material

\begin{tabular}{|c|c|c|c|c|c|c|}
\hline \multirow{2}{*}{$10^{-2} \mathrm{~S} / \mathrm{m}$} & \multicolumn{2}{|c|}{$f=10^{4} \mathrm{~Hz}$} & \multicolumn{2}{|c|}{$\mathrm{f}=10^{5} \mathrm{H} \mathrm{z}$} & \multicolumn{2}{|c|}{$\mathrm{f}=10^{6} \mathrm{H} \mathrm{z}$} \\
\hline & IZs! & $\operatorname{Arg}(Z s)$ & $|Z s|$ & $\operatorname{Arg}(Z s)$ & $|Z s|$ & $\operatorname{Arg}\left(Z_{s}\right)$ \\
\hline FDTD $(z=60 \mathrm{~m})$ & 1.57 & 84.61 & 10.13 & 50.40 & 28.10 & 44.45 \\
\hline $\begin{array}{l}\text { Theory } \\
\text { (multi-layer) }\end{array}$ & 1.57 & 84.01 & 10.13 & 50.46 & 28.10 & 44.88 \\
\hline $\begin{array}{l}\text { Theory } \\
\text { (infinite) }\end{array}$ & 2.81 & 45.00 & 8.89 & 45.00 & 28.10 & 45.00 \\
\hline $\begin{array}{l}10^{-3} \mathrm{~S} / \mathbf{m} \\
\text { FDTD } \\
(\mathrm{z}=60 \mathrm{~m})\end{array}$ & 1.58 & 89.97 & 15.68 & 83.45 & 99.40 & 47.13 \\
\hline $\begin{array}{l}\text { Theory } \\
\text { (multi-layer) }\end{array}$ & 1.58 & 89.40 & 15.68 & 84.00 & 102.87 & 48.69 \\
\hline $\begin{array}{l}\text { Theory } \\
\text { (infinite) }\end{array}$ & 8.89 & 45.00 & 28.10 & 45.00 & 88.86 & 45.00 \\
\hline
\end{tabular}

nications Laboratory of the University of Illinois [23]. The solution space consisted of 4000 cells $(n x=1, n y=100$, $n z=40$ ). The model took approximately $15 \mathrm{~min}$ to run on a DEC alpha computer work station.

The upper-half volume is air and the lower-half is the lossy earth with a conductivity of $0.01 \mathrm{~S} / \mathrm{m}$. Fig. 2 illustrates the solution space and the configuration of the source field $H_{x}$. An $H_{x}$ source was activated in the time domain using a Blackman-Harris time window (10\% cut off at $\left.10^{7} \mathrm{~Hz}\right)$ [23], and was located at coordinates $(x=0-1, y=0-100$, $z=35-35$ ). This configuration provided the best near source performance as judged by the surface impedance. Fig. 3 illustrates the variation in the amplitude and phase of the surface impedance $Z_{s}$ across the solution space for the two different source configurations. At grazing incidence, and with the source at $y=5$ cells, the region of uniform propagation extends from $y=50$ to 90 cells. This equates to an accurate result at a distance of $\lambda_{0} / 5000$ from the source, where $\lambda_{0}$ is the free space wavelength at the longest wavelength used. At normal incidence the entire earth plane gives accurate surface impedance values.

The surface impedance from a horizontally layered earth can be calculated analytically using a transmission line analogy [8]. The analytical solution for the surface impedance of this two layer model of a lossy earth over a perfect conductor was calculated using (2), and is given in Table I together with the FDTD results. Agreement is very good even though the frequency inequality (5) was violated slightly. Note that for a conductivity of $0.01 \mathrm{~S} / \mathrm{m}$ at $1 \mathrm{MHz}$, the multilayered result is identical to the infinite depth result because the skin depth is sufficiently small in comparison with the $20 \mathrm{~m}$ depth of lossy ground.

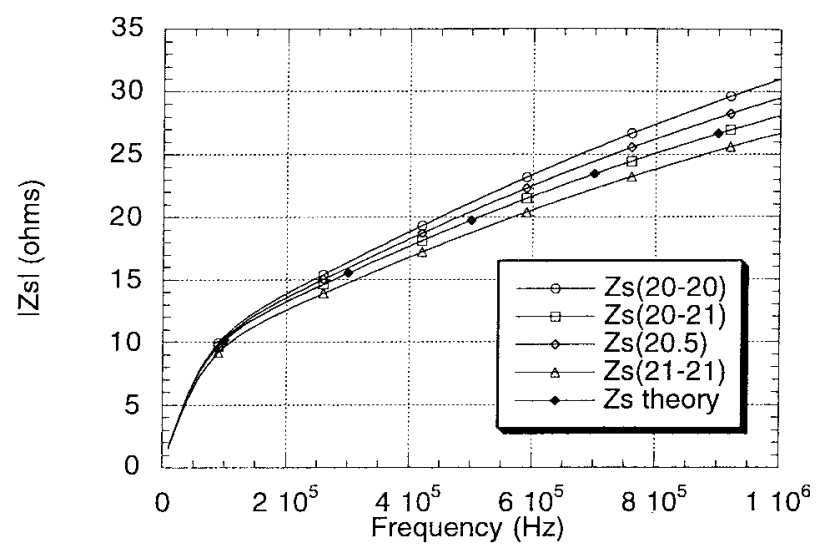

(a)

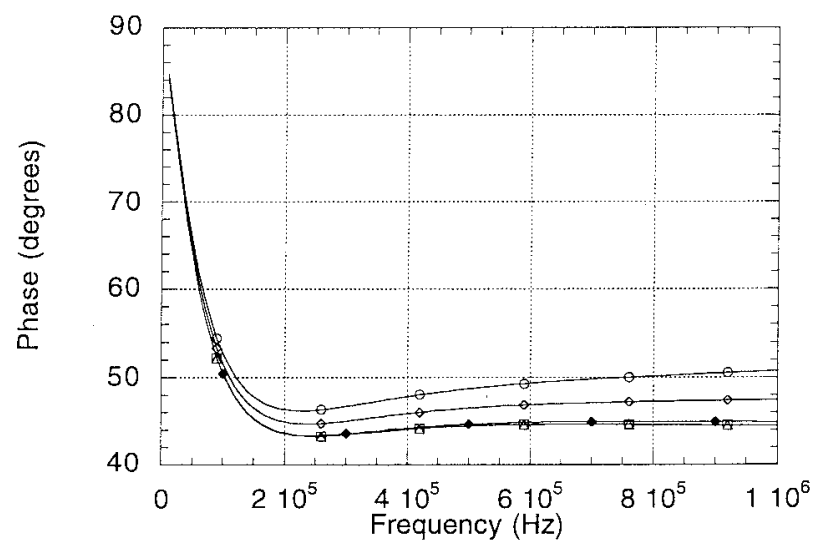

(b)

Fig. 4. Variation in surface impedance with frequency for a number of combinations of height indexes. Note that the index 20-21 refers to $E_{y}(20) / H_{x}(21)$, and 20.5 indicates that the impedance was calculated using the $H_{x}$ component averaged between two adjacent positions. ( $N=10000$, $y=70$ ). The earth structure is described in Fig. 3 .

Modeling was repeated for the grazing incidence case using a smaller number of air cells above the surface while leaving the remaining parts of the model unchanged. Reducing the number of air cells to five, which is equivalent to a spacing of $5 \mathrm{~m}$, gives a total solution space of $20 \times 25 \times 100$ cells. This model resulted in a slight increase in the distance from the source where the surface impedance stabilized at the correct theoretical value.

Fig. 4 shows the variation in $Z_{s}$ with grid position both above and below the surface of the lossy material $(\sigma=0.01$ $\mathrm{S} / \mathrm{m})$. In the Yee gridding [15] the $H$-field grid is displaced by one half cell from the $E$-field grid. $Z_{s}(20-21)$ refers to the impedance calculated using $E_{y}$ at $z=20$ and $H_{x}$ at $z=21$. $Z_{s}$ (20.5) was calculated using $E_{y}$ at $z=20$, and averaging the magnetic field values at $z=20$ and $z=21$. It is clear that the most accurate results are obtained using the $E_{y}$ component at $z=20$ and the $H_{x}$ component at $z=21$. This is results from the off-set numbering of the cells where there is only an $E$ field boundary at the first solution plane identified as $n=0$. Following Yee's technique [15], the electric field plane is used to derive the adjacent plane $(n=1) H$ cell values located one half a cell size above. Note that $n$ is the index used to label the planes. Thus, at the surface of the earth, the electric field is 


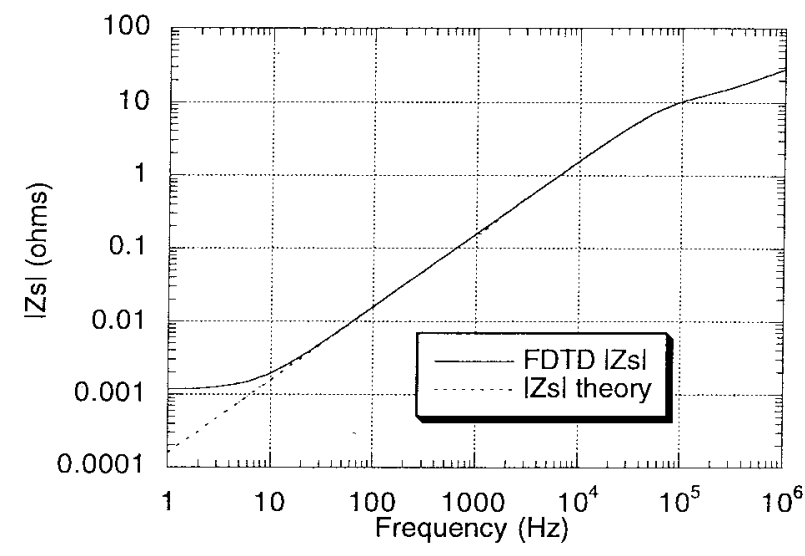

(a)

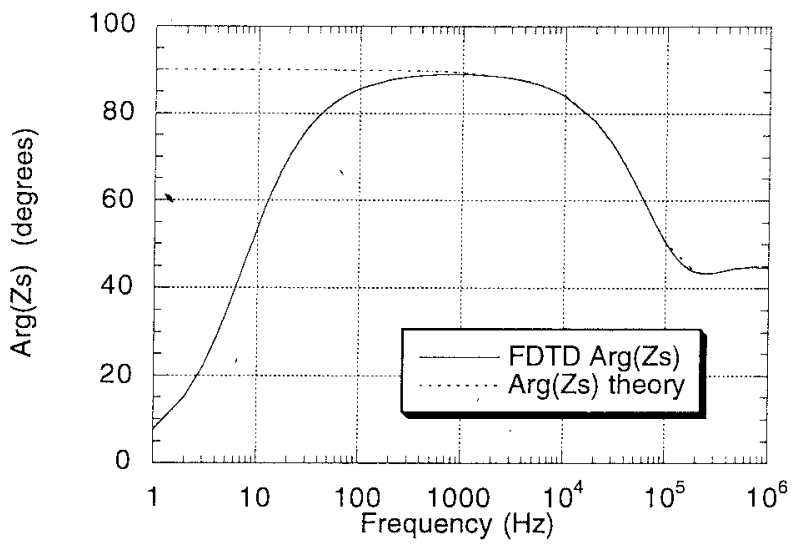

(b)

Fig. 5. Broad frequency characteristics of the FDTD surface impedance (continuous line) $(N=10000, z=70, \sigma=0.01 \mathrm{~S} / \mathrm{m})$ plotted together with the theoretical results (dotted line)

$n=20$, whereas the derived $H$ field is located at $n=21$. All FDTD surface impedance values were calculated using these two values; i.e., $E_{y}$ at the interface and $H_{x}$ displaced by one half cell above it.

Fig. 5 shows the accuracy of the model over a broad frequency range. Clearly, there are limitations on the model at both high frequencies, attributed to the fact that the cell size of the ground is too large, and at low frequencies due to the close proximity of the source to the measurement location. When this data is compared to that given in Fig. 1, it is clear that the inequality (5) can be taken as a guide rather than an absolute restriction. In Fig. 5, the magnitude of the surface impedance is accurate across a much broader frequency range when compared to the surface impedance phase angle. This was observed to be the case for most other situations throughout this study. For this model, results are accurate at frequencies greater than approximately $1 \mathrm{kHz}$.

The analytical solution for the surface impedance change across an abrupt change in earth conductivity was developed by d'Erceville and Kunetz [24] and for a dike by Rankin [25]. Using the same source configuration given in Fig. 2, the conductivity of the ground was abruptly changed from $\sigma=0.01 \mathrm{~S} / \mathrm{m}$ at $y=0-50 \mathrm{~m}$ to $\sigma=0.001 \mathrm{~S} / \mathrm{m}$ at $y=51-100 \mathrm{~m}$. The results for the FDTD model of the surface impedance across this change is given in Fig. 6, together with

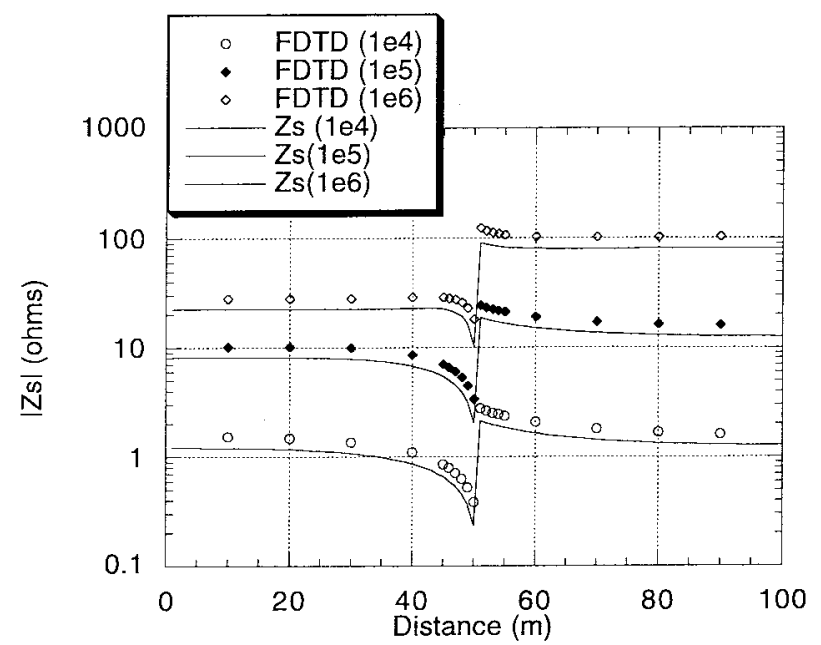

(a)

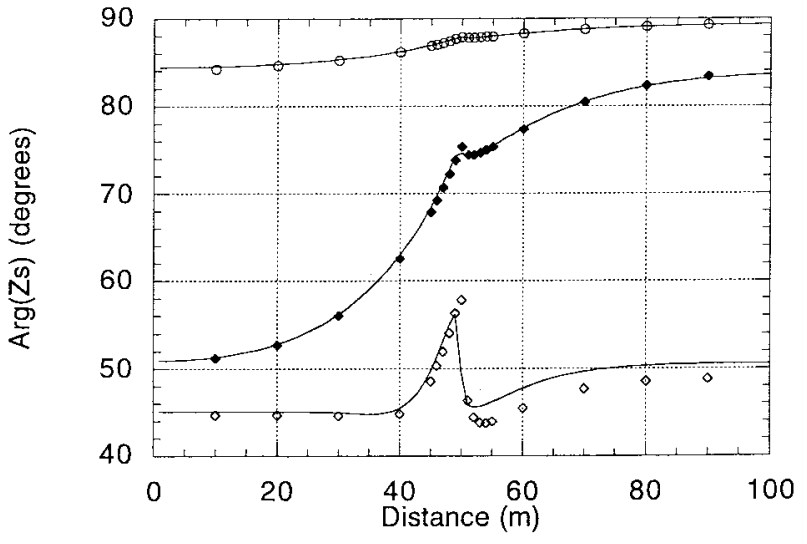

(b)

Fig. 6. Variation of surface impedance across an abrupt change in earth conductivity $(\sigma=0.01-0.001 \mathrm{~S} / \mathrm{m}$ at $y=50) .(N=10000)$. The theoretical result from Rankin [25] is plotted as a continuous line.

those obtained from an analytically derived series expansion [25]. While the two results are quite similar, it was found that the series expansion approach and the transmission line expression yield slightly different results. The FDTD results at the earth's surface away from the discontinuity were found to agree exactly with the transmission line calculation method for uniform horizontally layered earth planes shown in Table I [8]. One must conclude that the series formulation from Rankin for ULF frequencies has some inaccuracy at higher frequencies.

\section{THREE DimensionAL MODEL}

The 3-D model investigated was a small, vertically oriented conductive discontinuity in a uniform earth plane. The discontinuity extended down to the conductive basement. This represents an artificial geophysical target such as a water filled mine shaft or a steel cased drill hole which are common at old mine workings. This was analyzed using the FDTD code TAP [23]. The solution space consisted of $40 \times 100 \times 40$ cells with the following boundary conditions applied:

$$
\begin{aligned}
& x=0 \text { and } x=40 \\
& y=0, z=0, \text { and } y=100 \\
& z=40
\end{aligned}
$$

perfect magnetic boundary; perfect electric boundary; absorbing boundary conditions (second order Mur). 


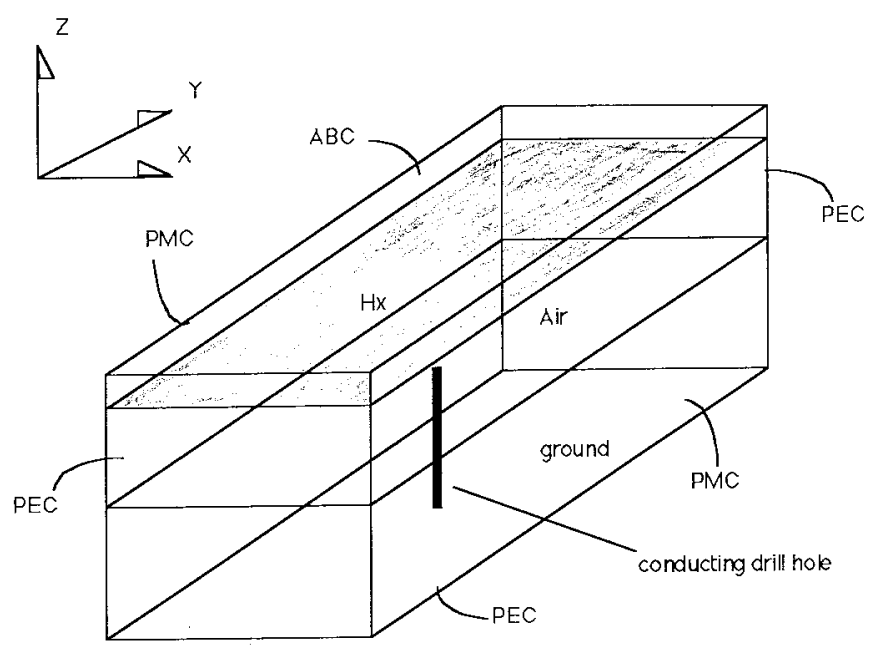

Fig. 7. Schematic diagram of the 3-D solution space for a vertical conductive shaft $(n x=40, n y=100 n z=40)$.

The upper-half volume is air and the lower half is the lossy earth plane $(\sigma=0.01 \mathrm{~S} / \mathrm{m})$. The $H_{x}$ source was located at coordinates $(x=0-40, y=0-100, z=35)$. The model took approximately four hours to run on a DEC alpha work station.

The variations of the surface impedance were calculated across the surface of a lossy earth half space (conductivity $0.01 \mathrm{~S} / \mathrm{m}$ ) containing a vertical conductive water filled shaft located at $x=20-21 \mathrm{~m}$ and $y=50-51 \mathrm{~m}$. The shaft reaches the conductive bedrock (e.g., an aquifer) at a depth of $20 \mathrm{~m}$ and has dimensions of $1 \times 1 \mathrm{~m}$ in cross section (i.e., one cell). The model is illustrated in Fig. 7, and is similar to other models used in RMT investigations [7]. It is important to note that while such a 3-D structure will require a surface impedance tensor for a full description of the interaction, the surface impedance technique used in this frequency range employ scalar surface impedance measurements only. These scalar measurements are made with the electric field sensor lying in the plane of incidence of the radiation. Thus, a set of scalar surface impedance measurements across the surface of the earth in the vicinity of the shaft measured in the $z$ direction is given in Fig. 8. It is clear that there is a significant anomaly in the vicinity of the shaft that is confined to within approximately $3 \mathrm{~m}$ of the surface opening. Note that in the FDTD model both the electric and magnetic fields are zero at the surface of the top of the shaft. The surface impedance at this point has been interpreted as being equal to zero.

It is significant that the surface impedance values are accurate to within $2 \%$ of the theoretical value less than 3 $\mathrm{m}$ from the anomaly. Thus problems associated with the proximity of the model boundaries are not significant.

\section{DISCUSSIONS AND CONCLUSIONS}

The FDTD technique has been successfully employed in modeling the electromagnetic surface impedance of lossy earth structures at frequencies above $500 \mathrm{~Hz}$. A 2-D algorithm with grid dimensions of $\lambda_{0} / 30000 \times \lambda_{0} / 750 \times \lambda_{0} / 300$ accurately modeled the surface impedance of a two layered earth (lossy earth above a perfect conductor) across the frequency range

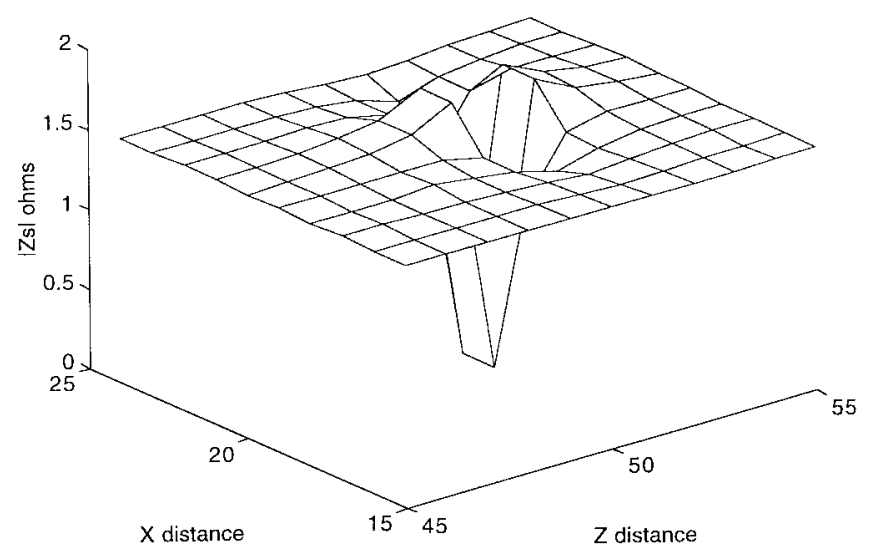

(a)

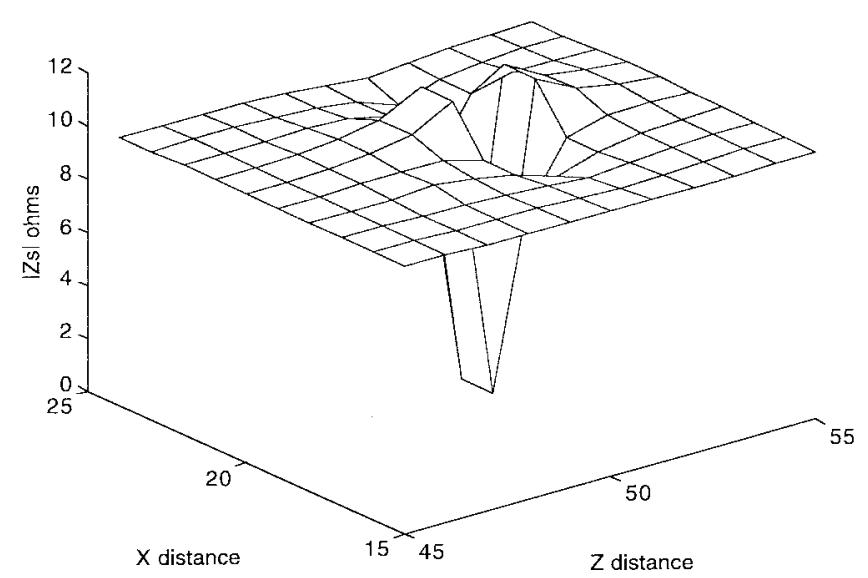

(b)

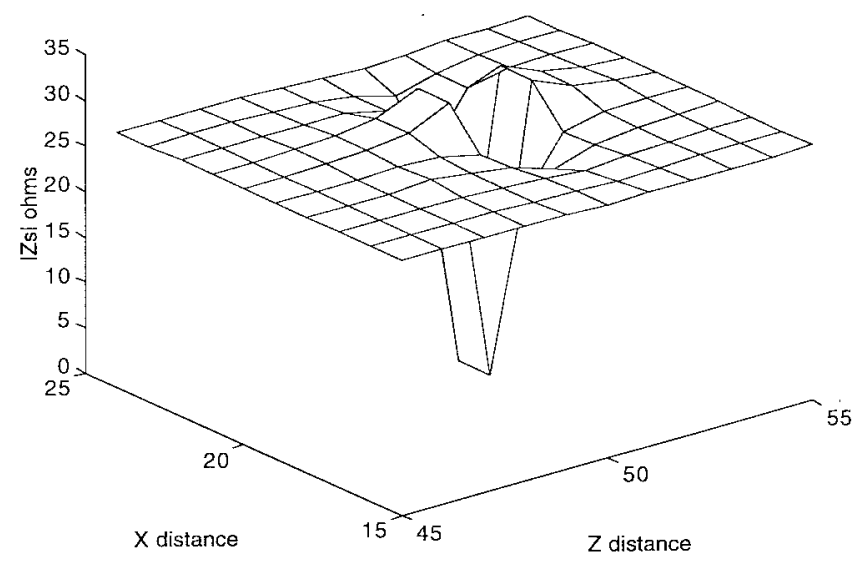

(c)

Fig. 8. (a) Variation in surface impedance magnitude in the vicinity of a vertical conductive shaft located at $x=20-21$ and $y=50-51$ at $10^{4} \mathrm{~Hz}$ ( $\left.N=6000, \sigma_{1}=0.01 \mathrm{~S} / \mathrm{m}\right)$. (b) Variation in surface impedance magnitude in the vicinity of a vertical conductive shaft located at $x=20-21$ and $y=50-51$ at $10^{5} \mathrm{~Hz}\left(N=6000, \sigma_{1}=0.01 \mathrm{~S} / \mathrm{m}\right)$. (c) Variation in surface impedance magnitude in the vicinity of a vertical conductive shaft located at $x=20-21$ and $y=50-51$ at $10^{6} \mathrm{~Hz}\left(N=6000, \sigma_{1}=0.01 \mathrm{~S} / \mathrm{m}\right)$.

$10_{4}-10_{6} \mathrm{~Hz}$. The surface impedance in this model was found to be accurate at distances greater than $\lambda_{0} / 500$ from the source field aperture when grazing incidence is used. However, normal incidence allows even closer source separation distances allowing for a smaller grid. In addition, the size of the model was minimized by careful choice of the cell size of 
the lossy medium for the frequency range of interest and an asymmetric location of the source aperture so it is close to an absorbing boundary. The surface impedance is most accurately calculated from the electric field component at the surface and the magnetic field one half cell above the surface.

The 3-D model used for the vertically oriented conductive water filled shaft is clearly much larger than needed as the scalar surface impedance perturbation is not significant at a distance of more than $3 \mathrm{~m}$ away from the surface location of the hole.

The FDTD method of modeling has been shown to offer the advantages of multi frequency analysis from a single model, the accurate modeling of the surface impedance throughout the surface of the earth in the model, and an ability to yield accurate results for quite small model sizes. The method, however, only yields satisfactory results at frequencies above approximately $500 \mathrm{~Hz}$.

\section{ACKNOWLEDGMENT}

D. V. Thiel wishes to express his sincere appreciation for the opportunity to visit the Electromagnetic Communications Laboratory at the University of Illinois for six months when this work was undertaken. The hospitality of all those working in the laboratory during the visit made for a very rewarding experience.

\section{REFERENCES}

[1] L. Cagniard, "Basic theory of the magnetotelluric method of geophysical prospecting," Geophysics, vol. 18, no. 3, pp. 605-635, July 1953.

[2] A. N. Tickhonov, "Determination of the electrical characteristics of deep strata of the earth's crust," Dok. Akad. Nauk., USSR, vol. 73, no. 2, pp. 295-297, 1950.

[3] Y. Kato and T. Kikuchi, "On the phase difference of earth current induced by the changes of the earth's magnetic field," Sci. Rep. Tohoku Univ. Serv. V. Geophys., vol. 2, pp. 139-145, 1950.

[4] S. J. Garner and D. V. Thiel, "Broad band VLF surface impedance measurements using atmospherics," in Western Pacific Geophys. Meet. AGU, Brisbane, Australia, July 1996.

[5] M. A. Goldstein and D. W. Strangway, "Audio-frequency magnetotellurics with a grounded electric dipole source," Geophysics, vol. 40, pp. 669-683, 1975.

[6] L. S. Collett and O. G. Jensen, Eds., "Geophysical applications of surface wave impedance measurements," Geolog. Surv. Can., 1982, Paper no. 8115

[7] A. Dupis, A. Choquier, and G. Bossuet, "La radio magneto-tellurique; une nouvelle methode de la geophysique appliquee," Bull. Soc. Geol. France, vol. 166 , no. 2, pp. 231-242, 1995 .

[8] J. R. Wait, Electromagnetic Waves in Stratified Media, 2nd ed. New York: Pergamon, 1969.

[9] G. Porstendorfer, Principles of Magnetotelluric Prospecting. Berlin, Germany: Gebruder Borntraeger, 1975.

[10] P. Silvester and C. R. S. Haslam, "Magnetotelluric modeling by the finite element method," Geophys. Prospect., vol. 4, no. 2, pp. 872-891, 1972.

[11] I. K. Reddy, D. Rankin, and R. J. Phillips, "Three-dimensional modeling in magnetotelluric and magnetic variational sounding," Geophys. J. R. Astron. Soc., vol. 51, pp. 313-315, 1977.

[12] P. Weidelt, "Electromagnetic induction in three-dimensional structures," J. Geophys., vol. 41, pp. 85-109, 1975.

[13] S. C. Ting and G. W. Hohmann, "Integral equation modeling of three-dimensional magnetotelluric response," Geophysics, vol. 46, pp. 182-197, 1981.

[14] G. W. Hohmann, "Three-dimensional em modeling," Geophys. Surv., vol. 6 , pp. 27-53, 1983.

[15] K. S. Yee, "Numerical solution of initial boundary value problems involving Maxwell's equations in isotropic media," IEEE Trans. Antennas Propagat., vol. AP-14, pp. 302-307, 1966.
[16] R. Luebbers, D. Steich, and K. Kunz, "FDTD calculation of scattering from frequency-dependent materials," IEEE Trans. Antennas Propagat., vol. 41, pp. 1249-1257, Sept. 1993

[17] K. S. Kunz and R. J. Luebbers, The Finite Difference Time Domain Method for Electromagnetics. Boca Raton, FL: CRC, 1993.

[18] W. L. Ko and R. Mittra, "Extremely low frequency modeling in lossy media using the FDTD," in IEEE AP-S Nat. Meet., Newport Beach, CA, 1995.

[19] T. R. Madden and R. L. Mackie, "Three-dimensional magnetotelluric modeling and inversion," Proc. IEEE, vol. 77, no. 2, pp. 318-333, Feb. 1989.

[20] R. L. Mackie, T. R. Madden, and P. E. Wannamaker, "Three-dimensional magnetotelluric modeling using difference equations-Theory and comparisons to integral equation solutions," Geophysics, vol. 58, no. 2, pp. 215-226, Feb. 1993.

[21] T. Wang and G. W. Hohmann, "A finite-difference, time-domain solution for three-dimensional electromagnetic modeling," Geophysics, vol. 58, no. 6, pp. 797-809, June 1993.

[22] G. Mur, "Absorbing boundary conditions for the finite-difference approximation of the time-domain electromagnetic field equations," IEEE Trans. Electromag. Compat., vol. EMC-23, pp. 377-382, Nov. 1981.

[23] M. A. Schamberger, TAP 3.25 Time-Domain Analysis Program for Solving Maxwell's Equations: Reference Manual. Urbana, IL: Univ. of Illinois Press, 1994.

[24] I. d'Erceville and G. Kunetz, "The effect of a fault on the earth's natural electromagnetic field," Geophysics, vol. 27, no. 5, pp. 651-655, 1962.

[25] D. Rankin, "The magneto telluric effect on a dike," Geophysics, vol 27, no. 5, pp. 666-676, 1962.

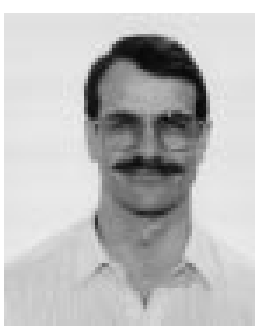

David V. Thiel (M'81-SM'88) received the B.Sc. degree (with honors) in physics from the University of Adelaide, Australia, in 1970, and the M.Sc. and Ph.D. degrees from James Cook University, Queensland, Australia, in 1974 and 1980, respectively.

$\mathrm{He}$ is currently a Professor in the School of Microelectronic Engineering at Griffith University, Queensland, and is also Director of the Radio Science Laboratory at the same institution. His current research interests include surface impedance measurements in electromagnetic geophysics and ice covered regions, numerical modeling techniques in electromagnetics, and antenna design.

Dr. Thiel is a member of the Wave Propagation Standards Definitions Committee of the IEEE Antennas and Propagation Society.

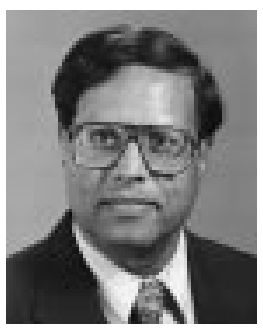

Raj Mittra (S'54-M'57-SM'69-F'71-LF'96) is a Professor of Electrical Engineering and a Senior Research Scienctist at the Applied Research Laboratory, Pennsylvania State University, University Park. He was previously the Director of the Electromagnetic Communication Laboratory of the Electrical and Computer Engineering Department and Research Professor of the Coordinated Science Laboratory, University of Illinois, Urbana. He has been a Visiting Professor at Oxford University, Oxford, U.K., and at the Technical University of Denmark, Lyngby. He is President of RM Associates, which is a consulting organization providing services to several industrial and governmental organizations, both in the United States and abroad. His professional interests include the areas of electromagnetic modeling and simulation of electronic packages, communication antenna design including GPS, broadband antennas, EMC analysis, radar scattering, frequency selective surfaces, microwave and millimeter wave integrated circuits, and satellite antennas. He has published approximately 400 journal papers and 20 books or book chapters on various topics related to electromagnetics.

Dr. Mittra is a Past-President of the IEEE Antennas and Propagation Society and he served as the Editor of the IEEE TRANSACTIONS ON ANTENNAS AND Propagation. He won the Guggenheim Fellowship Award in 1965 and the IEEE Centennial Medal in 1984. 